\title{
Constraints and Field Equations for Ten Dimensional Super Yang-Mills Theory ${ }^{\dagger}$
}

\author{
J. Harnad ${ }^{1 \star}$ and S. Shnider ${ }^{2}$ \\ 1 Département de Mathématiques Appliquées, Ecole Polytechnique, C.P. 6079, Succ. "A", Montréal, \\ Qué. CANADA H3C 3A7 \\ 2 Department of Mathematics, McGill University, Montreal, Canada, and Department of Mathema- \\ tics, Ben Gurion University, Beersheva 84105, Israel
}

\begin{abstract}
We give a complete proof of the equivalence between constraint equations and field equations for the $d=10, N=1$ supersymmetric Yang-Mills theory, a result proposed and partially proved recently by Witten [1]. Our approach explicitly reconstructs the superconnection satisfying the constraints from the on shell component fields. A key ingredient of the method is the choice of a suitable family of gauges, effectively eliminating all gauge dependence on anti-commuting co-ordinates. As a corollary, obtained by dimensional reduction, we also deduce the equivalence of constraints and field equations for the $d=4, N=4$ theory, as well as for $d=6, N=2$.
\end{abstract}

\section{Introduction}

The purpose of the present work is to supplement some recent results of Witten's [1] concerning the relationship between the superconnection constraint equations and the supersymmetric Yang-Mills equations in ten dimensions. As pointed out in $[1,2]$, a natural set of constraint equations involves the vanishing of the supercurvature along super null lines. This gives rise to the super twistor correspondence, in which bundles $\widetilde{E} \rightarrow \tilde{M}$ over super Minkowski space $\tilde{M}$, with a superconnection which is integrable along super null lines, correspond to certain bundles over the super ambitwistor space $\widetilde{A}$, whose points are the super null lines in $\tilde{M}$. These bundles are characterized by the fact that they are trivial over certain quadrics $Q \subset \tilde{A}$ corresponding to the set of super null lines through the same point of $\tilde{M}$. The importance of such a construction lies in the fact that for a suitable choice of the superspace extension $\tilde{M}$ of the underlying Minkowski space $M$, the constraint equations, together with the Bianchi identities for the supercurvature, imply the

\footnotetext{
$\dagger$ Research supported in part by the National Science and Engineering Research Council of Canada and the Israel Ministry of Science

* On leave of absence from: Department of Pure and Applied Mathematics, Stevens Institute of Technology, Hoboken NJ 07030, USA
} 
superfield Yang-Mills equations, as was shown for the maximally extended $(N=4)$ super Yang-Mills theory in four dimensions in the work of Sohnius [3] and Witten [2]. It was moreover generally presumed, following ideas formulated by Witten [2], that the passage from the constrained superconnection to the on shell Yang-Mills fields is one to one; that is, given any set of fields satisfying the supersymmetric YangMills equations, it is possible to construct a unique superconnection, satisfying the constraint equations, to which these fields correspond. Only in such a case is the supertwistor construction a full characterization of the supersymmetric Yang-Mills equations. A complete proof of this one to one equivalence for the $d=4, N=3$ case was actually only constructed recently [4]. The equivalence of the $N=3$ and $N=4$ field theory suggests that the corresponding result must also hold for $N=4$, although for this case an additional linear constraint on the curvature needs to be added $[2,3]$.

For the $d=10, N=1$ case, Witten has proved that for a suitably chosen (10|16) dimensional superspace extension $\tilde{M}$, the null-line integrability conditions imply the full set of field equations [1]. (Partial results of this nature were earlier demonstrated by Wess [5].) Since a suitable dimensional reduction procedure leads to both the field equations [6] and the constraints [7] for the $d=4, N=4$ theory, it is reasonable to conclude that the correspondence between these data for the $d=10$, $N=1$ theory is again one to one.

Developing methods used previously for the $d=4, N=3$ case [4], we shall provide here a complete proof of this one to one equivalence. As a corollary obtained through dimensional reduction, we shall have proved the $d=4, N=4$ equivalence as well as the corresponding result for the $d=6, N=2$ theory [7-10]. The logic of the demonstration is exactly the same as in [4]. It turns out, however, that the detailed computations are actually much simpler for the ten dimensional case than for the four dimensional one.

In principal, there are three separate sets of data which must be proved pairwise equivalent:

(i) The superconnection on $\widetilde{E} \rightarrow \tilde{M}$, satisfying the constraint equations.

(ii) The superfields satisfying the superfield equations.

(iii) The component fields satisfying the 10-dimensional supersymmetric YangMills equations.

In Sect. 2, we give a summary of the part of the result already proved by Witten; namely, the derivation of the superfield equations from the constraint equations. This is relatively straightforward once the content of the constraint equations together with the Bianchi identities has been analyzed. In order next to establish an invertible correspondence between (ii) and (iii), it is necessary to eliminate all gauge degrees of freedom depending on the anti-commuting superspace co-ordinates. To accomplish this, we introduce in Sect. 3 a special gauge condition particularly adapted to the embedding $M \hookrightarrow \tilde{M}$ of ordinary (10-dimensional) Minkowski space in its superspace extension. We call this the "DD-gauge" condition, defined by setting equal to zero the component of the connection along the Euler vector field $\mathscr{D}$ transverse to the embedding $M \hookrightarrow \tilde{M}$ under the standard linear splitting of even and odd co-ordinates. We deduce the action of the $\mathscr{D}$ operator on all superfields and thereby show how to reconstruct the superfields from their leading components and 
the superconnection from the superfields. This also allows us to deduce the action of the supersymmetry transformations upon the component fields. It remains to verify that, given any set of on-shell component fields, the superfields so constructed satisfy the appropriate field equations and the resulting superconnection satisfies the constraint equations. These statements are proved in Sect. 4 by induction on the degree of homogeneity in a componentwise expansion; i.e. the eigenvalues of $\mathscr{D}$. In fact, the inductive proof of the implication (iii) $\rightarrow$ (ii) involves a computation equivalent to the verification that the supersymmetry transformations really leave the field equations invariant. Similarly, the inductive proof of the implication (ii) $\rightarrow$ (i) involves a computation equivalent to the verification that the supersymmetry transformations close on shell to define a representation of the superalgebra. The fact that we actually have exhausted all possible consequences of the constraint equations follows from the invertibility of the maps (i)↔(ii) (iii) within the $\mathscr{D}$-gauge.

Finally, in Sect. 5 we show how a suitable splitting of $M$ into the sum of a four dimensional and a six dimensional space, together with the corresponding reduction of the isometry groups leads, through dimensional reduction, either to the standard $d=4, N=4$ superconnection constraints and field equations, or those for $d=6$, $N=2$ case. The equivalence of these data for each case follows as a corollary from the $d=10, N=1$ result.

\section{Superconnection Constraint Equations $\Rightarrow$ Super Field Equations}

In most of what follows, we shall be considering the complex version of the theory, although reality conditions are easily imposed at the end. The complexified super Minkowski space considered is of dimension (10|16), with standard co-ordinates $\left\{x^{\mu}, \theta^{A}\right\}_{\mu=0, . .9, A}=1, \ldots 16$ consisting of commuting Cartesian co-ordinates $\left\{x^{\mu}\right\}$ and anti-commuting spinorial co-ordinates $\left\{\theta^{A}\right\}$. An upper $A$ index refers to a basis for the fundamental 16-dimensional representation of the $\operatorname{Spin}(10, \mathbb{C})$ group, which is an irreducible component of the standard $32=16+16^{*}$ dimensional representation associated to the Clifford algebra, the other component $16^{*}$ being the contragredient representation. A lower $A$ index refers to the dual basis. No invariant quadratic (or symplectic) form exists since the 16 and 16* are inequivalent, and hence one cannot raise and lower indices. However, each representation is virtually real (i.e. invariant under an antilinear involution), which is what allows one to impose Majorana-type reality conditions [6]. The $\Gamma$ matrices in this representation map the 16 and $16^{*}$ into each other, and thus have the off diagonal block decomposition:

$$
\tilde{\Gamma}^{\mu}=\left[\begin{array}{c:c}
0 & \Gamma^{\mu C D} \\
\hdashline \Gamma_{A B}^{\mu} & 0
\end{array}\right] .
$$

Similarly, the quadratic elements of the Clifford algebra have the decomposition:

$$
\tilde{\Sigma}^{\mu \nu}=\left[\begin{array}{cc}
\Sigma_{B}^{\mu \nu A} & 0 \\
0 & -\Sigma_{D}^{t \mu \nu C}
\end{array}\right],
$$

where

$$
\Sigma_{B}^{\mu \nu A}=\frac{1}{2}\left[\Gamma^{\mu A C} \Gamma_{C B}^{v}-\Gamma^{v A C} \Gamma_{C B}^{\mu}\right] .
$$


We may define a frame using either the infinitesimal left translations $\left\{\partial_{\mu}, q_{A}^{L}\right\}$ or the right translations $\left\{\partial_{\mu}, q_{A}^{R}\right\}$.

$$
\begin{aligned}
& \partial_{\mu}=\frac{\partial}{\partial x^{\mu}}, \\
& q_{A}^{L}=\frac{\partial}{\partial \theta^{A}}+\Gamma_{A B}^{\mu} \theta^{B} \frac{\partial}{\partial x^{\mu}}, \\
& q_{A}^{R}=\frac{\partial}{\partial \theta^{A}}-\Gamma_{A B}^{\mu} \theta^{B} \frac{\partial}{\partial x^{\mu}} .
\end{aligned}
$$

Each set generates a representation of the superalgebra, with non-vanishing brackets:

or

$$
\begin{aligned}
& \left\{q_{A}^{L}, q_{B}^{L}\right\}=2 \Gamma_{A B}^{\mu} \partial_{\mu}, \\
& \left\{q_{A}^{R}, q_{B}^{R}\right\}=-2 \Gamma_{A B}^{\mu} \partial_{\mu},
\end{aligned}
$$

and the two sets commute with each other. We shall adopt the convention of expressing the null line integrability conditions and superfield equations in terms of the set $\left\{\partial_{\mu}, q_{A}^{L}\right\}$, while regarding the set $\left\{\partial_{\mu}, q_{A}^{R}\right\}$ as the supersymmetry generators.

Now, given a connection on a bundle ${ }^{1} \widetilde{E} \rightarrow \tilde{M}$ defined in some gauge by a 1 form $\omega$ on $\tilde{M}$ with values in the gauge algebra, the covariant derivative operator along any vector $X$ is:

$$
\mathscr{D}_{X} \equiv X+\omega(X)
$$

In particular, we denote:

$$
\mathscr{D}_{\partial_{\mu}} \equiv \mathscr{D}_{\mu} \equiv \partial_{\mu}+\omega_{\mu}
$$

and

$$
\mathscr{D}_{q_{A}^{L}} \equiv Q_{A} \equiv q_{A}^{L}+\omega_{A},
$$

where $\omega_{\mu} \equiv \omega\left(\partial_{\mu}\right), \omega_{A} \equiv \omega\left(q_{A}^{L}\right)$.

The constraint equations are:

$$
\left\{Q_{A}, Q_{B}\right\}=2 \Gamma_{A B}^{\mu} \mathscr{D}_{\mu} .
$$

According to Witten's twistor-type formulation [1] of $d=10, N=1$ supersymmetric Yang-Mills theory, their interpretation is the vanishing of curvature on super null lines. As such, they are the compatibility conditions for the integrability of the equations for a covariant constant section:

$$
\begin{aligned}
\lambda^{\mu} \mathscr{D}_{\mu} V & =0, \\
\lambda^{\mu} \Gamma_{\mu}^{A B} Q_{B} V & =0,
\end{aligned}
$$

1 A bundle over superspace can be thought of as a locally free sheaf of modules of type $(n \mid m)$ over the sheaf of superfunctions. A choice of gauge is a choice of local basis for the module. There are $n$ even sections and $m$ odd sections 
where $V: \tilde{M} \rightarrow \mathbb{C}^{n \mid m}$ represents a section of the bundle $\tilde{E}$ on which our super connection is operating, and $\lambda^{\mu} \lambda_{\mu}=0$. Defining the spinor superfield:

$$
\psi^{B} \equiv \frac{1}{10} \Gamma^{\mu A B}\left[\mathscr{D}_{\mu}, Q_{A}\right]
$$

and bosonic curvature:

$$
F_{\mu \nu}=\left[\mathscr{D}_{\mu}, \mathscr{D}_{\nu}\right]
$$

it follows from (2.7), together with the Bianchi identities, that the covariant derivative operator $Q_{A}$ acts as follows on $\mathscr{D}_{\mu}$, the bosonic part of the connection, $\psi^{B}$ and $F_{\mu \nu}$ respectively:

$$
\begin{aligned}
{\left[Q_{A}, \mathscr{D}_{\mu}\right] } & =-\Gamma_{\mu A B} \psi^{B}, \\
Q_{A} \psi^{B} & =+\frac{1}{2} \Sigma^{\mu \nu B}{ }_{A} F_{\mu \nu}, \\
Q_{A} F_{\mu \nu} & =\Gamma_{\mu A B} \mathscr{D}_{\nu} \psi^{B}-\Gamma_{\nu A B} \mathscr{D}_{\mu} \psi^{B} .
\end{aligned}
$$

These relations were all derived in [1] by Witten. They follow from the definitions in an elementary way with the help of a number of identities satisfied by the $\Gamma$-matrices which we list here:

$$
\begin{aligned}
& \Gamma^{\mu A B}=\Gamma^{\mu B A}, \quad \Gamma_{A B}^{\mu}=\Gamma_{B A}^{\mu}, \\
& \Gamma^{\mu A B} \Gamma_{B C}^{v}+\Gamma^{v A B} \Gamma_{B C}^{\mu}=2 g^{\mu v} \delta_{C}^{A}, \\
& \Gamma_{A B}^{\mu} \Gamma_{\mu C D}+\Gamma_{A C}^{\mu} \Gamma_{\mu B D}+\Gamma_{A D}^{\mu} \Gamma_{\mu B C}=0, \\
& \Gamma_{A B}^{\mu} \Gamma_{\mu}^{A C}=10 \delta_{B}^{C} . \\
& \Gamma_{A B}^{\mu} \Gamma_{v}^{A B}=16 \delta_{v}^{\mu}, \\
& \Sigma_{B}^{\mu \nu A} \Gamma_{v A C}=-9 \Gamma_{B C}^{\mu}, \\
& \sum_{B}^{\mu \nu A} \Gamma_{v}^{B C}=+9 \Gamma^{\mu A C}, \\
& \Gamma_{A B}^{\sigma} \Sigma_{C}^{\mu \nu B}+\Gamma_{B C}^{\sigma} \Sigma_{A}^{\mu \nu B}=-2 g^{\sigma \nu} \Gamma_{A C}^{\mu}+2 g^{\sigma \mu} \Gamma_{A C}^{v}, \\
& \Sigma_{B}^{\mu v A} \Gamma^{\sigma B C}+\sum_{B}^{\mu \nu C} \Gamma^{\sigma B A}=2 g^{v \sigma} \Gamma^{\mu A C}-2 g^{\mu \sigma} \Gamma^{v A C} \text {, } \\
& \Gamma^{\mu C B} \Gamma_{\mu A D} \Gamma^{\nu E D} \Gamma_{\nu F B}=-4 \Gamma^{\mu C E} \Gamma_{\mu F A}+12 \delta_{F}^{C} \delta_{A}^{E} \\
& +8 \delta^{C}{ }_{A} \delta_{F}^{E} \text {. }
\end{aligned}
$$

Equation (2.10b) can be derived using the fact that $Q_{A} \psi^{B}=\Sigma^{\mu \nu B}{ }_{A} \chi_{\mu \nu}$ for some $\chi_{\mu v}$, which follows from the Bianchi identity involving $Q_{A}, Q_{B}, D_{\mu}$.

Actually, only identities (2.11a, b, d, f, h, i, j) are needed in deriving (2.10a, b, c), but they are all useful in the computations to follow. Now, expressing:

$$
\Gamma_{A B}^{\mu} \mathscr{D}_{\mu}=\frac{1}{2}\left(Q_{A} Q_{B}+Q_{B} Q_{A}\right)
$$

and applying it to $\psi^{B}$, using $(2.10 \mathrm{a}, \mathrm{b}, \mathrm{c})$ for each derivation by $Q_{A}$ or $Q_{B}$, and the identity $(2.11 \mathrm{~g})$, we find the superfield Dirac equation:

$$
\Gamma_{A B}^{\mu} \mathscr{D}_{\mu} \psi^{B}=0 \text {. }
$$


Finally, applying $\Gamma_{v}^{A C} Q_{C}$ to (2.13), using (2.10a, b) and the identities (2.11a, c, e, i), we find the superfield Yang-Mills equations:

$$
\mathscr{D}^{\mu} F_{\mu \nu}+\frac{1}{2} \Gamma_{\nu A B}\left\{\psi^{A}, \psi^{B}\right\}=0 .
$$

This completes our summary of Witten's derivation of the superfield equations from the constraints. In the following two sections, we shall be concerned with the inverse implication.

\section{The $\mathscr{D}$-Gauge: Reconstruction of Superfields and Superconnection}

To reconstruct the superfields and superconnection from any given set of leading component fields, it is necessary to eliminate the gauge freedom associated with the $\left\{\theta^{A}\right\}$ co-ordinates. Proceeding exactly as in [4], we do this by requiring that the superconnection vanish along the transverse Euler vector field defined by the operator:

$$
\begin{aligned}
\mathscr{D} & =\theta^{A} \frac{\partial}{\partial \theta^{A}}, \\
\text { i.e. } \omega(\mathscr{D}) & =\theta^{A} \omega_{A}=0 .
\end{aligned}
$$

(Geometrically this means that the horizontal lift of $\mathscr{D}$ is tangential to the local section defining the gauge. ${ }^{2}$ ) Any two gauges satisfying such a condition can be related by a transformation $g$ independent of the $\left\{\theta^{A}\right\}$ variables, since (3.2) for each choice of gauge implies: $\mathscr{D} g=0$, and hence $g$ is a homogeneous polynomial of degree zero in the $\left\{\theta^{A}\right\}$. It also follows that in such a gauge the covariant Euler operator equals the ordinary one:

$$
\mathscr{D}=\theta^{A} Q_{A}=\theta^{A} \frac{\partial}{\partial \theta^{A}} .
$$

Using this fact, we deduce from the constraint equation (2.7) and from (2.10a-c), that $\mathscr{D}$ acts as follows upon the superconnection, spinor superfield $\psi^{B}$ and curvature superfield $F_{\mu \nu}$ :

$$
\begin{aligned}
(1+\mathscr{D}) \omega_{B} & =2 \theta^{A} \Gamma_{A B}^{\mu} \omega_{\mu}, \\
\mathscr{D} \omega_{\mu} & =-\theta^{A} \Gamma_{\mu A B} \psi^{B}, \\
\mathscr{D} \psi^{B} & =+\frac{1}{2} \theta^{A} \sum^{\mu \nu B}{ }_{A} F_{\mu \nu}, \\
\mathscr{D} F_{\mu \nu} & =\theta^{A} \Gamma_{\mu A B} \mathscr{D}_{\nu} \psi^{B}-\theta^{A} \Gamma_{\nu A B} \mathscr{D}_{\mu} \psi^{B} .
\end{aligned}
$$

(Note that (3.7) is not an independent relation, but follows from (3.5).)

2 Given a superconnection in an arbitrary gauge, the $\mathscr{D}$-gauge is obtained by applying the transformation $g$ defined by the ODE:

$$
\theta^{A} \frac{\partial g}{\partial \theta^{A}}=\theta^{A} \omega_{A} g
$$

The existence of a unique solution with $\left.g\right|_{\theta^{A}}=1$ is immediate from a component-wise expansion in $\theta^{A}$ 
These relations are non-dynamic, but serve to determine the superfields $\left(\omega_{\mu}, \omega_{A}, \psi^{B}\right)$ uniquely from the leading components $\left(\stackrel{\circ}{\omega}_{\mu}, \dot{\psi}^{B}\right)$ in a $\left\{\theta^{A}\right\}$ expansion, since $\mathscr{D}$ is positive semidefinite with integer eigenvalues equal to the degree of homogeneity in $\left\{\theta^{A}\right\}$. (We have $\stackrel{\circ}{A}_{A}=0$ by the gauge condition 3.2.) Specifically, one can recover $\left(\omega_{\mu}, \psi^{B}\right)$ recursively from $\left(\dot{\omega}_{\mu}, \dot{\psi}_{B}\right)$ by repeated application of $(3.5)$ and (3.6), and then deduce $\omega_{B}$ from (3.4) by dividing each term in the expansion of the right-hand side by the suitable integer eigenvalue of $(1+\mathscr{D})$. The leading terms are:

$$
\begin{aligned}
\psi^{B} & =\stackrel{\circ}{\psi}^{B}+\frac{1}{2} \theta^{A} \Sigma_{{ }^{\mu \nu B} F_{\mu \nu}}+\cdots, \\
\omega_{\mu} & =\stackrel{\circ}{\omega}_{\mu}-\theta^{A} \Gamma_{\mu A B} \dot{\iota}^{B}+\cdots, \\
\omega_{B} & =\theta^{A} \Gamma_{A B}^{\mu} \stackrel{\circ}{\omega}_{\mu}-\frac{2}{3} \theta^{A} \theta^{C} \Gamma_{A B}^{\mu} \Gamma_{\mu C D} \stackrel{\circ}{ }^{C}+\cdots .
\end{aligned}
$$

The higher terms are easily computed, but will not be needed in what follows.

The relations (3.5) and (3.6) resemble the supersymmetry transformations, but should not be confused with them. They are to be regarded as the non-dynamical part of the constraint equations, which allow us to reconstruct the superfields and superconnection uniquely in terms of the leading components $\left(\dot{\omega}_{\mu}, \dot{\psi}^{B}\right)$. The supersymmetry transformations are:

$$
\begin{aligned}
& \delta \stackrel{\circ}{\omega}_{\mu} \equiv \alpha^{A} \delta_{A} \stackrel{\circ}{\omega}_{\mu}=-\alpha^{A} \Gamma_{\mu A B} \stackrel{\circ}{\psi}^{B}, \\
& \delta \dot{\psi}_{B} \equiv \alpha^{A} \delta_{A} \dot{\psi}_{B}=\frac{1}{2} \alpha^{A} \Sigma^{\mu \nu B}{ }_{A}^{\circ} \dot{\circ}_{\mu \nu}
\end{aligned}
$$

and are thus obtained formally from (3.5), (3.6) by replacing the superfields $\left\{\omega_{\mu}, \psi^{B}\right\}$ by their leading components $\left\{\dot{\omega}_{\mu}, \dot{\psi}^{B}\right\}$ and the super-space co-ordinates $\left\{\theta^{A}\right\}$ by the parameters $\left\{\alpha^{A}\right\}$ defining the transformation.

In fact, Eqs. (3.9a, b) may be deduced from (3.5), (3.6) by correct interpretation of their geometrical meaning. Recalling the definition (2.9a) of $\psi^{B}$ as a part of the supercurvature tensor, the supersymmetry transformation (3.9b) should be regarded as the leading term of the corresponding Lie derivative of the curvature with respect to the supersymmetry generator $X \equiv \alpha^{A} q_{A}^{R}$. Since left and right translations commute, we have:

$$
\left(\mathscr{L}_{X} \Omega\right)(Y, Z)=X(\Omega(Y, Z)),
$$

where $\Omega$ is the curvature 2 -form and $Y, Z$ are any pair of left translations. Thus, by definition:

$$
\begin{aligned}
\delta \dot{\psi}^{B} & =\frac{1}{10} \Gamma^{\mu A B}\left[\mathscr{L}_{X} \Omega\left(\dot{\circ}_{\mu}, q_{A}^{L}\right)\right] & & \text { by (2.9a) } \\
& =\left(\alpha^{A} q_{A}^{\circ} \psi^{B}\right) & & \text { by (3.10) } \\
& =\frac{1}{2} \alpha^{A} \Sigma^{\mu \nu B}{ }_{A}^{\circ} \dot{F}_{\mu \nu} & & \text { by (3.8b). }
\end{aligned}
$$

A similar computation for $\delta \stackrel{\circ}{\mu}_{\mu}$ yields (3.9a).

Although this provides an interpretation of the relations (3.9a, b), it does not yet justify regarding these as infinitesimal variations of the leading components of a superfield constructed from (3.5)-(3.7), since the relations (3.4)-(3.7) are not invariant under supersymmetry transformations for arbitrary superfields. This is what underlies the usual observation that such transformations do not define a 
representation of the superalgebra off shell [11]. We shall see however in the next section that the recursive relations (3.4)-(3.7) together with the field equations for the leading components $\left\{\stackrel{\circ}{\omega}_{\mu}, \dot{\leftrightarrow}^{B}\right\}$ are in fact fully equivalent to the constraint equations, and hence are supersymmetric on shell (up to a gauge transformation which does not affect the leading terms). This is what will justify regarding (3.9a, b) as transformations of leading components of a superfield on shell.

\section{Inductive Proof: Field Equations $\Rightarrow$ Constraint Equations}

Having constructed superfields $\left\{\omega_{\mu}, \omega_{A}, \psi^{B}\right\}$ from the leading component fields $\left\{\stackrel{\circ}{\omega}_{\mu}, \dot{\psi}^{B}\right\}$ through the procedure of the preceding section, we must now verify that the resulting connection does indeed satisfy the constraints, provided $\left\{\dot{\infty}_{\mu}, \psi^{B}\right\}$ satisfy the field equations:

$$
\begin{aligned}
\Gamma_{A B}^{\mu} \mathscr{D}_{\mu} \dot{\psi}^{B} & =0, \\
\mathscr{D}^{\mu} \stackrel{\circ}{F}_{\mu \nu} & =-\frac{1}{2} \Gamma_{\nu A B}\left\{\dot{\psi}^{A}, \dot{\psi}^{B}\right\} .
\end{aligned}
$$

We do this in three stages. First, we prove by induction on the homogeneity in $\left\{\theta^{A}\right\}$ that (4.1a, b), together with (3.5)-(3.7) imply the superfield equations (2.13), (2.14). The usual argument for this sort of result [11] is that the supersymmetry of $(4.1 \mathrm{a}, \mathrm{b})$, together with the closure of the algebra generated by transformations $(3.9 \mathrm{a}, \mathrm{b})$ on shell imply that the superfields defined by applying the operator $\exp \left\{\theta^{A} \delta_{A}\right\}$ formally to the leading components will necessarily satisfy the superfield equations. Our inductive proof will in effect justify this formal manipulation. Secondly, we shall prove, again inductively, that if $\omega_{\mu}$ and $\psi^{B}$ satisfy the superfield equations, the relations $(2.10 \mathrm{a}, \mathrm{b}, \mathrm{c})$ will be satisfied. The computation involved in the inductive step will be seen for this case to be formally equivalent to the closure of the superalgebra on shell. Finally, we show explicitly that relations $(2.10 \mathrm{a}-\mathrm{c})$ imply the constraint equation (2.7) in the $\mathscr{D}$-gauge.

I. Inductive Proof (iii) $\rightarrow$ (ii). The first step of the induction, corresponding to $\theta^{A}$ homogeneity zero is verified because of the leading component field equations $(4.1 \mathrm{a}, \mathrm{b})$. Now, assuming validity of the superfield equations up to order $n$ in $\theta^{A}$, we have, by application of the operator $\mathscr{D}$ to (2.13) and use of the recursions (3.5), (3.6), (3.7):

$$
\begin{aligned}
& \left.\mathscr{D}\left(\Gamma_{A B}^{\mu} \mathscr{D}_{\mu} \psi^{B}\right) \quad \text { (to order } n+1\right)=-\theta^{C} \Gamma_{A B}^{\mu} \Gamma_{\mu C D}\left\{\psi^{D}, \psi^{B}\right\}+\frac{1}{2} \theta^{C} \Gamma_{A B}^{\mu} \Sigma_{C}^{\sigma \tau B} \mathscr{D}_{\mu} F_{\sigma \tau}
\end{aligned}
$$

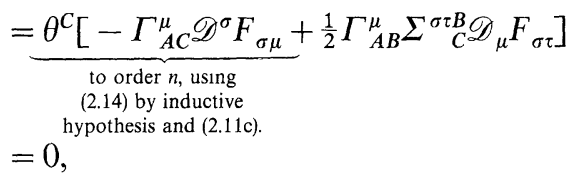

where the last step follows from the $\Gamma$-matrix identity $(2.11 \mathrm{~h})$ and the Bianchi identity:

$$
\mathscr{D}_{\mu} F_{\sigma \tau}+\mathscr{D}_{\tau} F_{\mu \sigma}+\mathscr{D}_{\sigma} F_{\tau \mu}=0
$$

Thus, Eq. (2.13) holds to order $n+1$ in $\theta^{A}$, since $\mathscr{D}$ is positive on this term. Similarly, 
applying $\mathscr{D}$ to the difference between the two sides of (2.14), and using the recursions (3.5)-(3.7),

$$
\begin{aligned}
\mathscr{D}[ & \left.\left.\mathscr{D}^{\mu} F_{\mu \nu}+\frac{1}{2} \Gamma_{\nu A B}\left\{\psi^{A}, \psi^{B}\right\}\right] \quad \text { (to order } n+1\right) \\
= & -\Gamma_{C B}^{\mu} \theta^{C}\left[\psi^{B}, F_{\mu \nu}\right]+\theta^{C} \mathscr{D}^{\mu}\left[\Gamma_{\mu C B} \mathscr{D}_{\nu} \psi^{B}-\Gamma_{\nu C B} \mathscr{D}_{\mu} \psi^{B}\right]+\frac{1}{2} \theta^{C} \Gamma_{\nu A B} \Sigma_{C}^{\sigma \tau A}\left[F_{\sigma \tau}, \psi^{B}\right] \\
= & -\Gamma_{C B}^{\mu} \theta^{C}\left[\left[\psi^{B}, F_{\mu \nu}\right]+\Gamma_{C B}^{\mu}\left[F_{\mu \nu}, \psi^{B}\right]\right. \\
& +\underbrace{\left.\frac{1}{2} \Gamma_{\nu C A} \Sigma^{\sigma \tau A}\left[F_{\sigma \tau}, \psi^{B}\right]+\frac{1}{2} \Gamma_{\nu A B} \Sigma^{\sigma \tau A}\left[F_{\sigma \tau}, \psi^{B}\right]\right]}_{\text {to order } n \text { by inductive hypothesis as explained below }} \\
= & 0 \quad \text { (by identity (2,11h)). }
\end{aligned}
$$

In the inductive step, we have used both the super-Dirac equation (2.13) (to order $n$ ) and its consequence:

$$
\mathscr{D}^{\mu} \mathscr{D}_{\mu} \psi^{B}=-\frac{1}{2} \sum_{C}^{\mu \nu B}\left[F_{\mu \nu}, \psi^{C}\right] .
$$

This completes the inductive proof of the superfield equations. Note that if we replaced all superfields by their leading components and $\mathscr{D} \rightarrow \delta, \theta^{A} \rightarrow \alpha^{A}$, the above computation would be identical to the verification that Eqs. $(4.1 \mathrm{a}, \mathrm{b})$ are invariant under the supersymmetry transformations $(3.8 \mathrm{a}, \mathrm{b})$.

II. Inductive Proof: Superfield Equations $\rightarrow$ Equations $(2.10 a-c)$. The first step of the induction follows by substituting the leading terms of the expansions $(3.8 \mathrm{a}, \mathrm{b}, \mathrm{c})$ into the relations $(2.10 \mathrm{a}, \mathrm{b})$ and verifying that the $\theta^{A}$-independent terms are equal.

$$
\begin{aligned}
& {\left[Q_{A}^{\circ}, \mathscr{D}_{\mu}\right]=\frac{\partial}{\partial \theta^{A}}\left(-\theta^{D} \Gamma_{\mu D C} \dot{\psi}^{C}+\cdots\right)=-\Gamma_{\mu A C} \dot{\psi}^{C},} \\
& {\left[Q_{A}^{\circ}, \psi^{B}\right]=\frac{\partial}{\partial \theta^{A}}\left(\frac{1}{2} \theta^{C} \Sigma_{C}^{\mu \nu B} \dot{F}_{\mu \nu}\right)=\frac{1}{2} \Sigma_{A}^{\mu \nu B} \stackrel{\circ}{F}_{\mu \nu} .}
\end{aligned}
$$

(Since (2.10c) is not independent of $(2.10 \mathrm{a}, \mathrm{b})$, but follows from (2.10a) by taking even covariant derivatives, it need not be considered separately.)

Next, we apply the operator $(1+\mathscr{D})$ to the difference between the two sides of $(2.10 \mathrm{a}, \mathrm{b})$, assuming these relations to be valid to order $n$ in $\theta^{A}$, and using the superfield equations, and the recursions (3.5)-(3.7),

$$
\begin{aligned}
(1 & \left.+\mathscr{D})\left[\left[Q_{A}, \mathscr{D}_{\mu}\right]+\Gamma_{\mu A B} \psi^{B}\right] \quad \text { (to order } n+1\right) \\
& =\left[2 \theta^{C} \Gamma_{A C}^{v}\left[\mathscr{D}_{v}, \mathscr{D}_{\mu}\right]+\theta^{C} Q_{A}\left(\Gamma_{\mu C B} \psi^{B}\right)+\frac{1}{2} \Gamma_{\mu A B} \theta^{C} \Sigma_{C}^{\sigma \tau B} F_{\sigma \tau}\right. \\
& =\theta^{C}[2 \Gamma_{A B}^{v} F_{v \mu}+\underbrace{\frac{1}{2} \Gamma_{\mu C B} \Sigma_{A}^{\sigma \tau B} F_{\sigma \tau}}_{\begin{array}{c}
\text { to order } n, \text { by inductive } \\
\text { hypothesis }
\end{array}}+\frac{1}{2} \Gamma_{\mu A B} \Sigma_{C}^{\sigma \tau B} F_{\sigma \tau}] \\
& =0 . \quad \text { (by identity }(2.11 \mathrm{~h}))
\end{aligned}
$$

Note that this computation is formally identical to the verification that the transformations $(3.9 \mathrm{a}, \mathrm{b})$ close under anti-commutation when applied to $\stackrel{\circ}{\mu}_{\mu}$, defining a representation of the superalgebra. The inductive hypothesis was used here, but not the superfield equations. However, the corresponding computation for $(2.10 b)$ requires the super-Dirac equation (2.13). Once again, applying $(1+\mathscr{D})$ to the 
difference between the two sides of (2.10b) and using (3.5)-(3.7),

$$
\begin{aligned}
(1 & \left.+\mathscr{D})\left[Q_{A} \psi^{B}-\frac{1}{2} \Sigma_{A}^{\mu \nu B} F_{\mu \nu}\right] \quad \text { (to order } n+1\right) \\
& =2 \theta^{C} \Gamma_{A C}^{\mu} \mathscr{D}_{\mu} \psi^{B}-\frac{1}{2} \theta^{C} \sum_{C}^{\mu \nu B} Q_{A} F_{\mu \nu}-\Sigma_{A}^{\mu \nu B} \theta^{C} \Gamma_{\mu C D} \mathscr{D}_{\nu} \psi^{B} \\
& =2 \theta^{C}[\Gamma_{A C}^{\mu} \mathscr{D}_{\mu} \psi^{B}-\underbrace{\sum_{\text {hypothesis }}^{\mu \nu B} \Gamma_{\mu A D} \mathscr{D}_{\nu} \psi^{D}}_{\text {to order } n \text { by inductive }}-\Sigma_{A}^{\mu \nu B} \Gamma_{\mu C D} \mathscr{D}_{\nu} \psi^{D}] \\
& =2 \theta^{C} \Gamma_{A C^{\prime}}^{\mu} \mathscr{D}_{\mu} \psi^{B}+\theta^{C}\left\{\Gamma^{\nu B E} \Gamma_{E A}^{\mu} \Gamma_{\mu C D}+\Gamma^{\nu B E} \Gamma_{E C}^{\mu} \Gamma_{\mu A D}\right\} \mathscr{D}_{\nu} \psi^{D} \\
& =2 \theta^{C} \Gamma_{A C}^{\mu} \mathscr{D}_{\mu} \psi^{D}-\theta^{C}\left\{\Gamma^{\nu B E} \Gamma_{E D}^{\mu} \Gamma_{\mu A C}\right\} \mathscr{D}_{\nu} \psi^{D} \quad \text { (by 2.11c) } \\
& =0 . \quad \text { (by identity }(2.11 \mathrm{~b}) \text { and the super-Dirac equation (2.13)). }
\end{aligned}
$$

Note again that this computation is formally identical to the verification that the transformations $(3.9 \mathrm{a}, \mathrm{b})$ close under anti-commutation when applied to $\psi^{B}$. This completes the inductive proof of Eqs. $(2.10 \mathrm{a}, \mathrm{b})$ (and also, by consequence $(2.10 \mathrm{c})$ ).

It now remains to verify that the superconnection constructed from (3.4)-(3.6) actually satisfies the constraint equation (2.7). To do this, we apply the positive operator $(2+\mathscr{D})$ to the difference between the two sides, giving:

$$
\begin{aligned}
(2+\mathscr{D})\left[\left\{Q_{A}, Q_{B}\right\}-2 \Gamma_{A B}^{\mu} \mathscr{D}_{\mu}\right]= & 2\left\{Q_{A}, Q_{B}\right\}+\left\{2 \theta^{C} \Gamma_{A C}^{\mu} \mathscr{D}_{\mu}, Q_{B}\right\}+\left\{Q_{A}, 2 \theta^{C} \Gamma_{B C}^{\mu} \mathscr{D}_{\mu}\right\} \\
& -2\left\{Q_{A}, Q_{B}\right\}-4 \Gamma_{A B}^{\mu} \mathscr{D}_{\mu}+2 \Gamma_{A B}^{\mu} \theta^{C} \Gamma_{\mu C D} \psi^{D} \\
= & +2 \theta^{C} \Gamma_{A C}^{\mu} \Gamma_{\mu B D} \psi^{C}+2 \theta^{C} \Gamma_{B C}^{\mu} \Gamma_{\mu A D} \psi^{D} \\
& \left.+2 \theta^{C} \Gamma_{A B}^{\mu} \Gamma_{\mu C D} \psi^{D} \quad \text { (by } 2.10 \mathrm{a}\right) \\
= & 0 . \quad(\text { by identity (2.11c)). }
\end{aligned}
$$

Therefore Eq. (2.7) holds, completing the demonstration.

\section{Dimensional Reduction to $d=4, N=4$ and $d=6, N=2$}

We now consider an orthogonal splitting $M=M_{4} \oplus M_{6}$ of complexified ten dimensional Minkowski space into the sum of a four dimensional subspace and a six dimensional one. The complex isometry group is $O(10, \mathbb{C})$, and it is convenient to express its representations, as well as those of the complex spin group $\operatorname{Spin}(10, \mathbb{C})$ in a basis adapted to this decomposition, with the corresponding reductions:

and

$$
O(10, \mathbb{C}) \supset O(4, \mathbb{C}) \times O(6, \mathbb{C})
$$

$$
\operatorname{Spin}(10, \mathbb{C}) \supset(\mathrm{Sl}(2, \mathbb{C}) \times \widetilde{\mathrm{Sl}}(2, \mathbb{C})) \times \operatorname{Sl}(4, \mathbb{C})
$$

Although we shall remain mainly in the complex setting, it is worth noting that the reality conditions which lead to the usual 4 and 6 dimensional structures involve the decompositions:

$$
O(1,9) \supset O(1,3) \times O(6, \mathbb{R})
$$

and

$$
\operatorname{Spin}(1,9) \supset \operatorname{SI}(2, \mathbb{C}) \times \operatorname{SU}(4)
$$


for the case where $M_{4}{ }^{\mathbb{R}} \subset M_{4}$ is Minkowskian and $M_{6}{ }^{\mathbb{R}} \subset M_{6}$ is Euclidean [6], or

$$
O(9,1) \supset O(4, \mathbb{R}) \times O(5,1)
$$

and

$$
\operatorname{Spin}(9,1) \supset(\mathrm{SU}(2) \times \widetilde{\mathrm{SU}}(2)) \times \mathrm{SU}^{*}(4)
$$

when $M_{4}^{\mathbb{R}}$ is Euclidean and $M_{6}^{\mathbb{R}}$ Minkowskian [7-10].

The 16-dimensional spinor representation under which the $\left\{\theta^{A}\right\}$ transform has the following decomposition relative to these reductions:

$$
16=4 \times 2+4 * \times \tilde{2}
$$

where 4 and $4 *$ are the fundamental representation of $\mathrm{Sl}(4, \mathbb{C})$ (or $\mathrm{SU}(4)$ or $\mathrm{SU}^{*}(4)$ ) and its dual, respectively, while 2 and $\tilde{2}$ represent the fundamental (undotted) representations of $\mathrm{Sl}(2, \mathbb{C})$ and $\widetilde{\mathrm{Sl}}(2, \mathbb{C})$, respectively. Under the first type of reality condition, we must identify $\widetilde{S l}(2, \mathbb{C})$ with the complex conjugate of $\operatorname{Sl}(2, \mathbb{C})$, and hence $\tilde{2}$ with the dotted $\operatorname{Sl}(2, \mathbb{C})$ spinor representation. Under the second type, the 2 and $\tilde{2}$ are identified as representations of the two different SU(2)'s. Denoting components relative to the standard basis in the 4 and $4 *$ respectively by an upper or lower Roman index $(i=1,2,3,4)$ and those for the standard basis in 2 and $\tilde{2}$ by $\{\alpha=0,1\},\{\dot{\alpha}=0,1\}$, (regardless of whether or not these are really complex conjugate representations), we may identify $\{A\}=\left\{(i \alpha),\left(\begin{array}{c}\dot{\alpha} \\ j\end{array}\right)\right\}$ and write the odd coordinates as

$$
\left\{\theta^{A}\right\}_{A=1, \ldots 16}=\left\{\theta^{i \alpha}, \theta_{j}^{\dot{\alpha}}\right\}_{i, j=1 \ldots 4 .} .
$$

Similarly, the 10-dimensional vector representation splits into the sum of an $O(4, \mathbb{C})$ vector, equivalent to $2 \times \tilde{2}$, for which we use the tensor product basis, with components labelled by a pair $\{\alpha \dot{\beta}\}$, and an $O(6, \mathbb{C})$ fundamental representation, equivalent to the bivector representation $4 \wedge 4 \simeq 4^{*} \wedge 4^{*}$ (this latter equivalence given by the volume form), with components labelled by an antisymmetric pair $(i j)=$ $-(j i)$. Thus, we identify $\{\mu\}=\{(\alpha \dot{\beta}),(i j)\}$ and write the even co-ordinates

$$
\left\{x^{\mu}\right\}_{\mu=\ldots 9}=\left\{x^{\alpha \hat{\beta}}, y^{i j}=-y^{j i}\right\},
$$

in terms of which the underlying complex metric is:

$$
d s^{2}=\operatorname{det}\left(d x^{\alpha \beta}\right)-\frac{1}{2} \varepsilon_{i j k l} d y^{i j} d y^{k l} .
$$

The reality condition corresponding to $(5.2 \mathrm{a})$ is given by

$$
x^{+}=x \quad \bar{y}=* y,
$$

where $(* y)^{i j} \equiv \frac{1}{2} \varepsilon^{i j k l} y^{k l}$. That corresponding to (5.3a) is given by:

$$
\bar{x}=\varepsilon x \varepsilon^{t} \quad \bar{y}=+T y T^{t}
$$

where

$$
T \equiv\left(\begin{array}{ll}
\varepsilon & 0 \\
0 & \varepsilon
\end{array}\right) \quad \varepsilon=\left(\begin{array}{rr}
0 & 1 \\
-1 & 0
\end{array}\right)
$$


Relative to these bases, we identify the nonvanishing components of the $\Gamma$-matrices as follows:

$$
\begin{aligned}
& \Gamma_{(i \beta)\left(\begin{array}{l}
\gamma \\
j
\end{array}\right)}^{\alpha \dot{\beta}}=\delta_{i}^{j} \delta_{\beta}^{\alpha} \delta_{\dot{\gamma}}^{\dot{\beta}}=\Gamma_{\left(\begin{array}{l}
\dot{\gamma} \\
j
\end{array}\right)_{(i \beta)}^{\alpha \dot{\beta}},}, \\
& \Gamma_{(i a)(j \beta)}^{k l}=\frac{1}{2} \varepsilon_{\alpha \beta}\left[\delta_{i}^{k} \delta_{j}^{l}-\delta_{j}^{k} \delta_{i}^{l}\right], \\
& \Gamma_{\left(\begin{array}{c}
\dot{\alpha} \\
i
\end{array}\right)\left(\begin{array}{c}
\dot{\beta} \\
j
\end{array}\right)}^{k}=\frac{1}{2} \varepsilon^{i j k l} \varepsilon_{\dot{\alpha} \dot{\beta}},
\end{aligned}
$$

for $\Gamma_{A B}^{\mu}$ and:

$$
\begin{aligned}
\Gamma^{\alpha \dot{\beta}(i \gamma)}\left(\begin{array}{l}
\dot{\gamma} \\
j
\end{array}\right) & =4 \delta_{j}^{i} \varepsilon^{\alpha \gamma} \varepsilon^{\dot{\beta} \dot{\gamma}}=\Gamma^{\dot{\alpha} \beta\left(\begin{array}{l}
\dot{\gamma} \\
j
\end{array}\right)(v \gamma)}, \\
\Gamma^{k l(i \alpha)(j \beta)} & =2 \varepsilon^{\alpha \beta} \varepsilon^{i j k l}, \\
\Gamma^{k(l}\left(\begin{array}{l}
\dot{\alpha} \\
i
\end{array}\right)\left(\begin{array}{l}
\dot{\beta} \\
j
\end{array}\right) & =2 \varepsilon^{\dot{\alpha} \dot{\beta}}\left[\delta_{i}^{k} \delta_{j}^{l}-\delta_{j}^{k} \delta_{i}^{l}\right]
\end{aligned}
$$

for $\Gamma^{\mu A B}$. The left translation generators on $\tilde{M}$ may then be identified as:

$$
\begin{aligned}
& \left(q_{A}^{L}\right)=\left(q_{i \alpha}, q_{\dot{\beta}}^{j}\right), \\
& \left(\partial_{\mu}\right)=\left(\partial_{\alpha \dot{\beta}}, \partial_{i j}\right),
\end{aligned}
$$

where

$$
\begin{aligned}
q_{i \alpha} & =\frac{\partial}{\partial \theta^{i \alpha}}+\theta_{i}^{\dot{\alpha}} \frac{\partial}{\partial x^{\alpha \dot{\alpha}}}+\varepsilon_{\alpha \beta} \theta^{i \beta} \frac{\partial}{\partial y^{i j}}, \\
q_{\dot{\alpha}}^{i} & =\frac{\partial}{\partial \theta_{i}^{\dot{\alpha}}}+\theta^{i \alpha} \frac{\partial}{\partial x^{\alpha \dot{\alpha}}}+\frac{1}{2} \varepsilon^{i j k l} \varepsilon_{\dot{\alpha} \dot{\beta}} \theta_{j}^{\dot{\beta}} \frac{\partial}{\partial y^{k l}}, \\
\partial_{\alpha \dot{\alpha}} & =\frac{\partial}{\partial x^{\alpha \dot{\alpha}}}
\end{aligned}
$$

satisfy the superalgebra relations

$$
\begin{aligned}
\left\{q_{i \alpha}, q_{j \beta}\right\} & =2 \varepsilon_{\alpha \beta} \partial_{i j}, \\
\left\{q_{\dot{\alpha}}^{i}, q_{\dot{\beta}}^{j}\right\} & =\varepsilon_{\dot{\alpha} \dot{\beta}} \varepsilon^{i j k l} \partial_{k l}, \\
\left\{q_{i \alpha}, q_{\beta}^{j}\right\} & =2 \delta_{j}^{i} \partial_{\alpha \beta} .
\end{aligned}
$$

Note that when acting upon functions independent of the $\left\{y^{i j}\right\}$ co-ordinates, the generators $\left\{q_{i \alpha}, q_{\beta}^{j}, \partial_{\alpha \beta}\right\}$ reproduce the $d=4, N=4$ superalgebra relations. Similarly, on functions independent of the $\left\{x^{\alpha \hat{\beta}}\right\}$, they reproduce the $d=6, N=2$ relations $^{3}$.

The covariant derivatives may similarly be decomposed as:

$$
\begin{aligned}
& \left(Q_{A}\right)=\left(Q_{i \alpha}, Q_{\dot{\beta}}^{j}\right), \\
& \left(\mathscr{D}_{\mu}\right)=\left(\mathscr{D}_{\alpha \dot{\beta}}, \mathscr{D}_{i j}\right),
\end{aligned}
$$

3 This is not really a precise characterization, since in this complex version the algebra has an internal symmetry $\mathrm{Sl}(2, \mathbb{C}) \times \mathrm{Sl}(2, \mathbb{C})$ covariance rather than just $\mathrm{Sl}(2, \mathbb{C})$. It may be regarded as a second complexification of the $N=2$, algebra, involving complex left and right spinors 
where

$$
\begin{aligned}
Q_{i \alpha} & =q_{i \alpha}+\omega_{i \alpha}, \\
Q_{\alpha}^{i} & =q_{\dot{\alpha}}^{i}+\omega_{\dot{\alpha}}^{i}, \\
\mathscr{D}_{\alpha \dot{\beta}} & =\partial_{\alpha \dot{\beta}}+A_{\alpha \dot{\beta}}, \\
\mathscr{D}_{i j} & =\partial_{i j}+W_{i j},
\end{aligned}
$$

and

$$
\begin{aligned}
& \left(\omega_{A}\right)=\left(\omega_{i \alpha}, \omega_{\dot{\alpha}}^{i}\right), \\
& \left(\omega_{\mu}\right)=\left(A_{\alpha \dot{\beta}}, W_{i j}\right) .
\end{aligned}
$$

In this notation, the constraint equation (2.7) may be expressed:

$$
\begin{aligned}
\left\{Q_{i \alpha}, Q_{j \beta}\right\} & =2 \varepsilon_{\alpha \beta} \mathscr{D}_{i j}, \\
\left\{Q_{\dot{\alpha}}^{i}, Q_{\dot{\beta}}^{j}\right\} & =\varepsilon_{\dot{\alpha} \dot{\beta}} \varepsilon^{i j k l} \mathscr{D}_{k l}, \\
\left\{Q_{i \alpha}, Q_{\dot{\beta}}^{j}\right\} & =2 \delta_{i}^{j} \mathscr{D}_{\alpha \dot{\beta}} .
\end{aligned}
$$

Similarly, the spinor superfield $\psi^{B}$ may be decomposed as:

$$
\left(\psi^{B}\right)=\left(\psi^{i \alpha}, \psi_{j}^{\beta}\right)
$$

where, defining

$$
\begin{aligned}
\chi_{i \dot{\alpha}} & \equiv \frac{1}{2} \varepsilon_{\dot{\alpha} \beta} \psi_{i}^{\dot{\beta}}, \\
\chi_{\alpha}^{i} & \equiv \frac{1}{2} \varepsilon_{\alpha \beta} \psi^{i \beta},
\end{aligned}
$$

Eq. (2.10a) may be re-expressed

$$
\begin{aligned}
{\left[Q_{i \alpha}, \mathscr{D}_{\beta \dot{\gamma}}\right] } & =\varepsilon_{\alpha \beta} \chi_{i \dot{\gamma}}, \\
{\left[Q_{\dot{\alpha}}^{i} \mathscr{D}_{\beta \dot{\gamma}}\right] } & =\varepsilon_{\dot{\alpha} \dot{\gamma}} \chi_{\beta}^{i}, \\
{\left[Q_{i \alpha}, \mathscr{D}_{j k}\right] } & =\varepsilon_{i j k l} \chi_{\alpha}^{l}, \\
{\left[Q_{\dot{\alpha}}^{i}, \mathscr{D}_{j k}\right] } & =\delta_{j}^{i} \chi_{k \dot{\alpha}}-\delta_{j}^{i} \chi_{j \dot{\alpha}} .
\end{aligned}
$$

Decomposing the curvature tensor

$$
\left(F_{\mu \gamma}\right)=\left(f_{\alpha \beta}, f_{\dot{\alpha} \dot{\beta}}, F_{\alpha \dot{\beta}, i j}, F_{i j, k l}\right)
$$

where

$$
\begin{aligned}
{\left[\mathscr{D}_{\alpha \dot{\alpha}}, \mathscr{D}_{\beta \dot{\beta}}\right] } & \equiv \varepsilon_{\alpha \beta} f_{\dot{\alpha} \dot{\beta}}+\varepsilon_{\dot{\alpha} \dot{\beta}} f_{\alpha \beta}, \\
{\left[\mathscr{D}_{\alpha \dot{\alpha}}, \mathscr{D}_{i j}\right] } & \equiv F_{\alpha \dot{\alpha}, i j}, \\
{\left[\mathscr{D}_{i j}, \mathscr{D}_{k l}\right] } & \equiv F_{i j, k l},
\end{aligned}
$$

we have, from Eq. (2.10b),

$$
\begin{aligned}
Q_{i \alpha} \chi_{j \dot{\alpha}} & =2 F_{\alpha \dot{\alpha}, j j}, \\
Q_{\alpha}^{i} \chi_{\alpha}^{j} & =\varepsilon^{i j k l} F_{\alpha \dot{\alpha}, k l},
\end{aligned}
$$




$$
\begin{aligned}
& Q_{i \alpha} \chi_{\beta}^{j}=2 \delta_{i}^{j} f_{\alpha \beta}+\frac{1}{2} \varepsilon_{\alpha \beta} \varepsilon^{j k l m} F_{l m, i k}, \\
& Q_{\dot{\alpha}}^{j} \chi_{i \beta}=2 \delta_{i}^{j} f_{\dot{\alpha} \dot{\beta}}-\frac{1}{2} \varepsilon_{\dot{\alpha} \dot{\beta}} \varepsilon^{j k l m} F_{l m, i k} .
\end{aligned}
$$

Equation (2.10c) may be similarly decomposed, giving the action of $\left(Q_{i \alpha}, Q_{\beta}^{j}\right)$ on each component of the curvature, but since this follows as a consequence of $(2.10 \mathrm{a}, \mathrm{b})$ through application of even derivations only, it need not be listed separately.

Decomposing the field equations (2.13), (2.14) in the basis, we obtain,

$$
\begin{aligned}
& \varepsilon^{\alpha \beta} \mathscr{D}_{\alpha \dot{\beta}} \chi_{\beta}^{i}+\frac{1}{2} \varepsilon^{i j k l} \mathscr{D}_{k l} \chi_{\dot{\beta}_{J}}=0, \\
& \varepsilon^{\dot{\beta} \dot{\gamma}} \mathscr{D}_{\beta \dot{\beta}} \chi_{l \dot{\gamma}}+\mathscr{D}_{i j} \chi_{\beta}^{j}=0,
\end{aligned}
$$

for the fermionic part, and

$$
\begin{aligned}
& \varepsilon^{\dot{\alpha} \beta} \mathscr{D}_{\gamma \dot{x}} f_{\beta \dot{\gamma}}+\varepsilon^{\alpha \beta} \mathscr{D}_{\dot{\alpha} \dot{\gamma}} f_{\beta \gamma}+\frac{1}{4} \varepsilon^{i j k l} \mathscr{D}_{k l} F_{\gamma \gamma, i j}+\left\{\chi_{\gamma}^{i}, \chi_{i \dot{\gamma}}\right\}=0, \\
& \varepsilon^{\alpha \beta} \varepsilon^{\dot{\alpha} \dot{\beta}} \mathscr{D}_{\alpha \dot{\alpha}} F_{\beta \dot{\beta}, l j}-\frac{1}{2} \varepsilon^{m n k l} \mathscr{D}_{m n} F_{k l, i j}+\frac{1}{2} \varepsilon^{\alpha \beta} \varepsilon_{i j k l}\left\{\chi_{\alpha}^{k}, \chi_{\beta}^{l}\right\}+\varepsilon^{\dot{\alpha} \dot{\beta}}\left\{\chi_{i \dot{\alpha}}, \chi_{j \dot{\beta}}\right\}=0 \text {, }
\end{aligned}
$$

for the bosonic part.

The $\mathscr{D}$-gauge condition (3.2) in our reduced basis takes the form

$$
\theta^{i \alpha} \omega_{i \alpha}+\theta_{i}^{\dot{\alpha}} \omega_{\dot{\alpha}}^{i}=0,
$$

and the $\mathscr{D}$ recursion relations (3.4)-(3.6) allowing us to reconstruct the superfields and superconnection from leading components become:

$$
\begin{aligned}
(1+\mathscr{D}) \omega_{i \alpha} & =2 \theta^{j \beta} \varepsilon_{\alpha \beta} W_{i j}+2 \theta_{i}^{\dot{\beta}} A_{\alpha \dot{\beta}}, \\
(1+\mathscr{D}) \omega_{\dot{\alpha}}^{i} & =2 \theta^{i \beta} A_{\beta \dot{\alpha}}+\varepsilon_{\dot{\alpha} \dot{\beta}} \varepsilon^{i j k l} \theta_{j}^{\beta} W_{k l}, \\
\mathscr{D} A_{\alpha \dot{\beta}} & =-\varepsilon_{\alpha \beta} \theta^{i \beta} \chi_{l \dot{\beta}}+\varepsilon_{\dot{\alpha} \dot{\beta}} \theta_{i}^{\dot{\alpha}} \chi_{\alpha}^{i}, \\
\mathscr{D} W_{i j} & =\varepsilon_{i j k l} \theta^{k \alpha} \chi_{\alpha}^{l}+\theta_{i}^{\alpha} \chi_{j \dot{\alpha}}-\theta_{j}^{\dot{\alpha}} \chi_{l \dot{\alpha}}, \\
\mathscr{D} \chi_{i \dot{\alpha}} & =2 \theta^{j \alpha} F_{\alpha \dot{\alpha}, j i}+2 \theta_{i}^{\dot{\beta}} f_{\dot{\alpha} \dot{\beta}}+\frac{1}{2} \varepsilon_{\dot{\alpha} \dot{\beta}} \theta_{j}^{\dot{\beta}} \varepsilon^{j k l m} F_{l m, i k}, \\
\mathscr{D} \chi_{\alpha}^{i} & =2 \theta^{i \beta} f_{\alpha \beta}-\frac{1}{2} \varepsilon_{\alpha \beta} \theta^{j \beta} \varepsilon^{i k l m} F_{l m, j k}+\theta_{j}^{\dot{\alpha}} \varepsilon^{j i k l} F_{\alpha \dot{\alpha}, k l} .
\end{aligned}
$$

The reality conditions corresponding to $(5.2 \mathrm{a}, \mathrm{b}),(5.7)$ may be extended to the superspace $\tilde{M}$ so as to preserve the super-algebra relations $(5.13 \mathrm{a}, \mathrm{b}, \mathrm{c})$ if we require:

$$
\begin{array}{ll}
\bar{\theta}^{i \alpha}=\theta_{i}^{\dot{\alpha}}, & \bar{\theta}_{i}^{\dot{\alpha}}=\theta^{i \alpha}, \\
\bar{q}_{i \alpha}=q_{\dot{\alpha}}^{i}, & \bar{q}_{\dot{\alpha}}^{i}=q_{i \alpha},
\end{array}
$$

where the bar indicates an extension to $\tilde{M}$ of the anti-linear involution (generalized complex conjugation) on $M$ underlying the reality condition (5.7). Correspondingly, the constraints and field equations will be preserved if the superconnection components and spinor superfield satisfy:

$$
\begin{aligned}
\sigma\left(\omega_{i \alpha}\right) & =\omega_{\dot{\alpha}}^{i}, \quad \sigma\left(\omega_{\alpha}^{i}\right)=\omega_{i \alpha}, \\
\sigma\left(A_{\alpha \beta}\right) & =A_{\beta \dot{\alpha},} \\
\sigma\left(W_{i j}\right) & =\frac{1}{2} \varepsilon_{i j k l} W_{k l}, \\
\sigma\left(\chi_{i \alpha}\right) & =-\chi_{\alpha}^{i}, \quad \sigma\left(\chi_{\alpha}^{i}\right)=-\chi_{i \dot{\alpha}},
\end{aligned}
$$


where $\sigma$ is the anti-linear involutive automorphism of the gauge algebra defining the particular real form considered, (e.g., $\sigma(\xi)=-\xi^{+}$if the gauge group is $U(n)$ ). Similarly, the reality conditions corresponding to $(5.3 \mathrm{a}, \mathrm{b}),(5.8)$ may be extended to $\tilde{M}$ by requiring:

$$
\begin{array}{ll}
\bar{\theta}^{i \alpha}=\varepsilon^{\alpha \beta} T_{j}^{i} \theta^{j \beta}, & \bar{\theta}_{i}^{\alpha}=\varepsilon^{\dot{\alpha} \dot{\beta}} T_{i}^{j} \theta_{j}^{\dot{\beta}}, \\
\bar{q}_{i \alpha}=\varepsilon_{\alpha \beta} q_{j \beta} T_{i}^{j}, & \bar{q}_{\dot{\alpha}}^{i}=\varepsilon_{\dot{\alpha} \dot{\beta}} T_{j}^{i} q_{\beta}^{j},
\end{array}
$$

where $\left(T_{j}^{i}\right)$ is the matrix defined in (5.9). The corresponding conditions on the superfields are:

$$
\begin{aligned}
\sigma\left(\omega_{i \alpha}\right) & =\varepsilon_{\alpha \beta} \omega_{j \beta} T_{i}^{j}, \quad \omega_{\dot{\alpha}}^{i}=\varepsilon_{\dot{\alpha} \beta} T_{j}^{i} \omega_{\beta}^{j}, \\
\sigma\left(A_{\alpha \beta}\right) & =\varepsilon_{\alpha \beta} \varepsilon_{\dot{\beta} \dot{\gamma}} A_{\beta \dot{\gamma}}, \\
\sigma\left(W_{i j}\right) & =T_{i}^{k} W_{k l} T_{j}^{l}, \\
\sigma\left(\chi_{\alpha}^{i}\right) & =-\varepsilon_{\alpha \beta} T_{j}^{i} \chi_{\beta}^{j}, \quad \sigma\left(\chi_{i \dot{\alpha}}\right)=-\varepsilon_{\dot{\alpha} \dot{\beta}} T_{i}^{j} \chi_{j \dot{\beta}} .
\end{aligned}
$$

Now, assuming the superconnection to be invariant under the six dimensional translation group generated by $\left\{\partial_{i j}\right\}$, we obtain the dimensional reduction to a (4|16) dimensional supermanifold $\tilde{M}_{4}$ defined as the quotient of $\tilde{M}$ by this action. All superfields derived above retain the same form, but become independent of the $\left\{y^{i j}\right\}$ co-ordinates. The covariant derivative operators $\mathscr{D}_{i j}$ reduce to Lie multiplication by the gauge algebra valued Lorentz scalar superfields $W_{i j}$ which, in view of the constraint equations $(5.17 \mathrm{a}, \mathrm{b})$ may be identified as the antisymmetric part of the curvature term $\left\{Q_{i \alpha}, Q_{j \beta}\right\}$ under the transposition $\{\alpha \leftrightarrow \beta\}$ :

$$
W_{i j}=-\varepsilon^{\alpha \beta}\left\{Q_{i \alpha}, Q_{j \beta}\right\} .
$$

The constraint equations then reduce to the usual form for the $d=4, N=4$ theory,

$$
\begin{aligned}
\left\{Q_{i \alpha}, Q_{j \beta}\right\}+\left\{Q_{i \beta}, Q_{j \alpha}\right\} & =0, \\
\left\{Q_{\dot{\alpha}}^{i}, Q_{\dot{\beta}}^{j}\right\}+\left\{Q_{\dot{\beta}}^{i}, Q_{\dot{\alpha}}^{j}\right\} & =0, \\
\left\{Q_{i \alpha}, Q_{\dot{\beta}}^{j}\right\} & =2 \delta_{i}^{j} \mathscr{D}_{\alpha \dot{\beta}}, \\
\varepsilon^{\alpha \beta}\left\{Q_{i \alpha}, Q_{j \beta}\right\} & =\frac{1}{2} \varepsilon_{i j k l} \varepsilon^{\dot{\delta} \beta}\left\{Q_{\dot{\alpha}}^{k}, Q_{\dot{\beta}}^{l}\right\} .
\end{aligned}
$$

The relations $(5.35 \mathrm{a}-\mathrm{c})$ represent the usual integrability conditions on super null lines in $\tilde{M}_{4}$, while (5.35d) is an additional linear vanishing condition on the curvature required by the dimensional reduction.

Since the dimensional reduction does not affect the anti-commuting coordinates $\left\{\theta^{A}\right\}$, all the relations $(5.20 \mathrm{a}-\mathrm{d}),(5.23 \mathrm{a}-\mathrm{c})$ remain valid in the reduced space, provided we make the appropriate substitutions:

$$
\begin{aligned}
\mathscr{D}_{i j} & \rightarrow\left[W_{i j},\right], \\
F_{\alpha \hat{\beta}, i j} & \rightarrow \mathscr{D}_{\alpha \dot{\beta}} W_{i j}, \\
F_{i j, k l} & \rightarrow\left[W_{i j}, W_{k l}\right] .
\end{aligned}
$$

The $\mathscr{D}$-recursion relations (5.27), (5.28), (5.29) also remain valid with these substitutions, allowing us again to uniquely reconstruct the reduced (4|16) 
dimensional superconnection from the leading component fields $\left\{\stackrel{\circ}{\alpha \beta}_{\alpha}, \stackrel{\circ}{W}_{i j}, \dot{\chi}_{i \dot{\alpha}},,_{\beta}^{j}\right\}$. The reduced form of the field equations implied by the constraints $(5.35 \mathrm{a}-\mathrm{d})$ may be read off from Eqs. (5.24a, b) and (5.25a, b) again by making the substitutions (5.36ac). Equations $(5.24 \mathrm{a}, \mathrm{b})$ reproduce the usual Dirac equations, with coupling to the scalar fields $\left\{W_{i j}\right\}$, while Eq. (5.25a) becomes the four dimensional super Yang-Mills equation and (5.25b) the superfield form of the scalar field equations. By restriction of our inductive argument to this case, we see that the full equivalence of field equations and constraints for the $d=4, N=4$ case follows as a corollary to the $d=10, N=1$ result.

Similarly, to obtain the $d=6, N=2$ reduction, we assume the superconnection to be invariant under the four dimensional translation group generated by $\left\{\partial_{\alpha \dot{\beta}}\right\}$. Quotienting $\tilde{M}$ by this action gives rise to the (6|16) dimensional supermanifold $\tilde{M}_{6}$ extending $M_{6}$. All superfields obtained by the previous construction become independent of the $\left\{x^{\alpha \hat{\beta}}\right\}$ co-ordinates, and the covariant derivative operators $\mathscr{D}_{\alpha \beta}$ reduce to Lie multiplication by the gauge algebra valued scalar superfields $A_{\alpha \beta}$. The latter, in view of the reduced form of the constraint equation $(5.17 \mathrm{c})$ may be defined as the trace part of the supercurvature term $\left\{Q_{i \alpha}, Q_{\beta}^{j}\right\}$ :

$$
A_{\alpha \dot{\beta}}=\frac{1}{8}\left\{Q_{i \alpha}, Q_{\dot{\beta}}^{i}\right\} \text {. }
$$

The constraint equations $(5.17 \mathrm{a}-\mathrm{c})$ then reduce to the correct form of the complex $d=6, N=2$ theory [7-10]:

$$
\begin{aligned}
& \left\{Q_{i \alpha}, Q_{j \beta}\right\}=2 \varepsilon_{\alpha \beta} \mathscr{D}_{i j}, \\
& \left\{Q_{\dot{\alpha}}^{i}, Q_{\dot{\beta}}^{j}\right\}=\varepsilon_{\dot{\alpha} \dot{\beta}} \varepsilon^{i j k l} \mathscr{D}_{k l}, \\
& \left\{Q_{i \alpha}, Q_{\dot{\beta}}^{j}\right\}-\frac{1}{4} \delta_{i}^{j}\left\{Q_{k \alpha}, Q_{\dot{\beta}}^{k}\right\}=0,
\end{aligned}
$$

which again are interpretable as integrability conditions along super null lines [9] in $\tilde{M}_{6}$.

The relations (5.20), (5.23) and the $\mathscr{D}$-recursions (5.27), (5.23) remain valid in the reduced space, as a consequence of $(5.38 \mathrm{a}-\mathrm{c})$, provided we make the substitutions:

$$
\begin{aligned}
\mathscr{D}_{\alpha \dot{\beta}} & \rightarrow\left[A_{\alpha \dot{\beta}},\right], \\
f_{\alpha \beta} & \rightarrow-\frac{1}{2} \varepsilon^{\dot{\alpha} \dot{\beta}}\left[A_{\alpha \dot{\alpha}}, A_{\beta \dot{\beta}}\right], \\
f_{\dot{\alpha} \dot{\beta}} & \rightarrow-\frac{1}{2} \varepsilon^{\alpha \beta}\left[A_{\alpha \dot{\alpha}}, A_{\beta \dot{\beta}}\right], \\
F_{\alpha \dot{\beta}, k l} & \rightarrow-\mathscr{D}_{k l} A_{\alpha \dot{\beta}} .
\end{aligned}
$$

The same substitutions reduce the field equations $(5.24 \mathrm{a}, \mathrm{b}),(5.25 \mathrm{a}, \mathrm{b})$ to those for the $d=6, N=2$ theory, with (5.25b) becoming the Yang-Mills equation with spinor and scalar sources, and (5.25a) the superfield form of the scalar field equations for $\left\{A_{\alpha \beta}\right\}$. Once again, the implications: constraints $\Rightarrow$ field equations are preserved by the reductions, since these do not affect the $\left\{\theta^{A}\right\}$ variables, and so are the inductive proofs of the implications: component field equations $\Rightarrow$ superfield equations $\Rightarrow$ constraint equations. Thus, the $d=6, N=2$ equivalence is also established as a corollary to the $d=10, N=1$ result.

Finally, the reality conditions discussed above may all be equally applied to the 
reduced spaces, the set $(5.2 \mathrm{a}, \mathrm{b}),(5.7),(5.20 \mathrm{a}, \mathrm{b})$ and $(5.31 \mathrm{a}-\mathrm{d})$ reproducing the usual four dimensional real Minkowski superspace results and the set (5.3a, b), (5.8), $(5.32 \mathrm{a}, \mathrm{b})$ and $(5.33 \mathrm{a}-\mathrm{d})$ those for the real six dimensional case.

Acknowledgements. We would like to thank E. Witten and J. Hurtubise for helpful discussions relating to the material presented in this paper.

\section{References}

1. Witten, E.: Twistor-like transform in ten dimensions. Princeton preprint (May 1985), to appear in Nucl. Phys. B (1986)

2. Witten, E.: An interpretation of classical Yang-Mills theory. Phys. Lett. 77B, 394 (1978)

3. Sohnius, M.: Bianchi identities for supersymmetric gauge theories. Nucl. Phys. B136, 461 (1978)

4. Harnad, J., Hurtubise, J, Légaré, M., Shnider, S.: Constraint equations and field equations in supersymmetric $N=3$ Yang-Mills theory. Nucl. Phys. B256, 609 (1985)

5. Wess, J.: Supersymmetric gauge theories. In: Unified Field Theories and Beyond, Proc. 5th Johns Hopkins Workshop, 5 (1981)

6. Brink, L., Scherk, J., Schwarz, J.: Supersymmetric Yang-Mills theories. Nucl. Phys. B121, 77 (1977)

7. Siegel, W.: Superfields in higher-dimensional spacetime. Phys. Lett. 80B, 220 (1979)

8. Howe, P. S., Sierra, G., Townsend, P. K.: Supersymmetry in six dimensions. Nucl. Phys. B221, 331 (1983)

9. Devchand, C.: Integrability on Lightlike Lines in Six Dimensional Superspace. Freiburg preprint (September 1985)

10. Koller, J.: A six-dimensional superspace approach to extended superfields. Nucl. Phys. B222, 319 (1983)

11. See, e.g. Gates, S. J., Jr., Grisaru, M. T., Rocek, M., Siegel, W.: Superspace. Reading, MA.: Benjamin/Cummings, 1983

Communicated by A. Jaffe

Received December 16, 1985 
